\title{
Polypropylene/Montmorillonite Nanocomposites. Review of the Synthetic Routes and Materials Properties
}

\author{
E. Manias,* A. Touny, L. Wu, K. Strawhecker, B. Lu, and T. C. Chung \\ Department of Materials Science and Engineering, The Pennsylvania State University, \\ 325-D Steidle Building, University Park, Pennsylvania 16802
}

Received February 12, 2001. Revised Manuscript Received May 21, 2001

\begin{abstract}
The synthetic routes and materials properties of polypropylene/montmorillonite nanocomposites are reviewed. The nanocomposite formation is achieved in two ways: either by using functionalized polypropylenes and common organo-montmorill onites, or by using neat/ unmodified polypropylene and a semi-fluorinated organic modification for the silicates. All the hybrids can be formed by solventless melt-intercalation or extrusion, and the resulting polymer/inorganic structures are characterized by a coexistence of intercalated and exfoliated montmorillonite layers. Small additions-typically less than 6 wt \%-of these nanoscale inorganic fillers promote concurrently several of the polypropylene materials properties, including improved tensile characteristics, higher heat deflection temperature, retained optical clarity, high barrier properties, better scratch resistance, and increased flame retardancy.
\end{abstract}

\section{Introduction}

The very large commercial importance of polypropylene (PP) has also been driving an intense investigation of PP composites reinforced by particulates, fibers, and layered inorganic fillers. ${ }^{1,2}$ In particular, in the case of layered inorganic fillers, talc and mica had been traditionally attracting the most interest. ${ }^{2}$ However, recent advances in polymer/clay and polymer/silicate nanocomposite materials ${ }^{3,4}$ have inspired efforts to disperse montmorillonite-based fillers in PP. ${ }^{5-12}$

Although it has been long known that polymers can be mixed with appropriately modified clay minerals and synthetic clays, ${ }^{13,14}$ the field of polymer/silicate nanocomposites has gained large momentum recently. Two were the major findings that pioneered the revival of these materials: First, the report of a nylon-6/montmorill onite material from Toyota research, ${ }^{15,16}$ where very moderate inorganic loadings resulted in concurrent and remarkable enhancements of thermal and mechanical properties. Second, Giannelis et al. found that it is possible to melt-mix polymers with clays without the use of organic solvents. ${ }^{17}$ Since then, the high promise for industrial applications has motivated vigorous research, which revealed concurrent dramatic enhancements of many materials properties by the nanodispersion of inorganic silicate layers. Where the property enhancements originate from the nanocomposite structure, these improvements are generally applicable across a wide range of polymers. ${ }^{3}$ At the same time, there were also discovered property improvements in these nanoscale materials that could not be realized by conventional fillers, as for example a general flameretardant characteristic ${ }^{18}$ and a dramatic improvement in barrier properties. ${ }^{19,20}$

Montmorillonite $(\mathrm{mmt})$ is a naturally occurring 2:1 phyllosilicate, which has the same layered and crystal-

\footnotetext{
* To whom correspondence should be addressed. E-mail: manias@ psu.edu.
}

line structure as talc and mica but a different layer charge. ${ }^{13,14}$ The mmt crystal lattice consists of 1-nmthin layers, with a central octahedral sheet of alumina fused between two external silica tetrahedral sheets (in such a way that the oxygens from the octahedral sheet also belong to the silica tetrahedra). I somorphic substitution within the layers (for example, $\mathrm{Al}^{3+}$ replaced by $\mathrm{Mg}^{2+}$ or $\mathrm{Fe}^{2+}$ ) generates a negative charge-defined through the charge exchange capacity (CEC)-and for $\mathrm{mmt}$ is typically $0.9-1.2$ mequiv/g depending on the mineral origin. These layers organize themselves in a parallel fashion to form stacks with a regular van der Waals gap between them, called interlayer or gallery. In their pristine form their excess negative charge is balanced by cations $\left(\mathrm{Na}^{+}, \mathrm{Li}^{+}, \mathrm{Ca}^{2+}\right)$ which exist hydrated in the interlayer. Obviously, in this pristine state mmt is only miscible with hydrophilic polymers, such as poly(ethylene oxide) and poly(vinyl alcohol).19,21 To render mmt miscible with other polymers, one must exchange the alkali counterions with cationic-organic surfactants, such as alkylammoniums. ${ }^{3,4}$

\section{Synthetic Routes and Nanocomposite Structures}

The Thermodynamic Challenge. In general, an interplay of entropic and enthal pic factors determines the outcome of whether an organically modified $\mathrm{mmt}$ (o-mmt) will be dispersed-intercalated or exfoliatedin a polymer. ${ }^{22-24}$ Dispersion of $\mathrm{mmt}$ in a polymer requires sufficiently favorable enthal pic contributions to overcome any entropic penalties. ${ }^{25}$ Favorable enthalpy of mixing for the polymer/o-mmt is achieved when the polymer/mmt interactions are more favorable compared to the surfactant/mmt interactions. ${ }^{22-24} \mathrm{~F}$ or most polar or polarizable polymers, an al kylammonium surfactant (the most commonly used organic modifica- 
tion) is adequate to offer sufficient excess enthal $p y^{26}$ and promote the nanocomposite formation. However, in the case of polypropylene, the alkylammonium-based o-mmt has surfactants with the same al iphatic-apolar-nature as PP. Consequently, such systems are at theta conditions, and there is no favorable excess enthalpy to promote PP/alkylammonium-mmt dispersion. Thus, the "challenge" with PP is to design systems where the polymer/mmt interactions are more favorable than the surfactant/mmt interactions.

There are two ways to overcome this challenge:

1. I mprove the interactions between the polymer and the mmt so as to become more favorable than the alkylsurfactant/mmt interactions. This can be achieved by PP "functionalization", that is, introducing polar or polarizable groups in the polymer.

2. Decrease the enthalpic interactions between the surfactant and the mmt, which effectively will render the PP/mmt contacts favorable. This second route is more challenging, as the alkyl-surfactant/mmt interactions are already very poor (that is exactly the reason these surfactants work so well in dispersing most nonaliphatic polymers). However, semi-fluorinated surfactants do have more unfavorable interactions than the hydrogenated polyol efins, and if used appropriately to organically modify the $\mathrm{mmt}$, they will promote PP/ommt miscibility.

Initial Efforts. Surprisingly, none of thesetwo routes was employed in the first studies aiming to devel op PP/ o-mmt materials. ${ }^{5-10}$ Instead, polypropylene ol igomers modified with either maleic anhydride (MA) or hydroxyl groups $(\mathrm{OH})$ were first mixed with octadecylammoniumexchanged montmorillonite, creating a master batch, which was subsequently blended with neat PP, usually assisted by strong mechanical shear in an extruder or mixer. In this way, the PP polymer and the MApolypropylene pretreated o-mmt are effectively at theta conditions, and the extrusion is only promoting mixing due to the effect of the mechanical shear. As a result, the structure and the properties of the resulting hybrid materials depended strongly on the processing conditions and ranged from very moderate dispersions and property improvements $s^{5-8}$ to good dispersions and better performing hybrids. ${ }^{10}$ Obviously, a MA-polypropylene pretreatment with very low maleic anhydride content did not promote the nanocomposite formation, ${ }^{5}$ and very high maleic anhydride content made the master batch so robust that mmt did not mix further with neat PP. 6,7

Nanocomposite Formation through PP Functionalization. The incorporation in the PP polymer of any functional group, which is attracted by the $\mathrm{mmt}$ surfaces to a greater degree than the methylene and methine, will promote PP miscibility with alkylammonium-modified mmt.22-24 To test this approach, random copolymers of PP with typically $1 \mathrm{~mol} \%$ of functionalized monomers were synthesized. The polymer characteristics are given in Table 1. All the functionalized PPs were derived from the same random PP copolymer synthesized by metallocene catalysis, which contained $1 \mathrm{~mol}$ $\%$ p-methylstyrene (p-MS) comonomers. Subsequently, the $\mathrm{p}-\mathrm{MS}$ 's were interconverted to functional groups containing hydroxyl $(\mathrm{OH})$ and maleic anhydride by lithiation and free-radical reactions, respectively. ${ }^{27}$ The
Table 1. Functionalized-PP Characteristics ${ }^{a}$

\begin{tabular}{|c|c|c|c|c|c|}
\hline & \multicolumn{2}{|c|}{ Functionalized PP: random copolymers } & $\mathrm{x}$ & $\mathrm{M}_{\mathrm{w}}$ & $\mathrm{T}_{\mathrm{m}}$ \\
\hline \multirow{2}{*}{$\begin{array}{l}\text { A: } \\
\text { B: }\end{array}$} & & PP-r- $(\text { PP-MS })_{x}$ & 1.0 & 200000 & 154 \\
\hline & & PP-r-(PP-MA $)_{x}$ & 0.5 & 200000 & 155 \\
\hline \multirow[t]{2}{*}{$\mathrm{c}$} & & PP-r- $(\mathrm{PP}-\mathrm{OH})_{\mathrm{x}}$ & 0.5 & 200000 & 155 \\
\hline & \multicolumn{2}{|c|}{ Functionalized PP: diblock copolymer } & & & \\
\hline \multirow{2}{*}{ D: } & $\left(\mathrm{CH}_{2}-\mathrm{CH}\right)\left(\mathrm{CH}_{2} \mathrm{C}^{-3}\right)$ & PP-b-(PMMA $)_{x}$ & 1.0 & 200000 & 155 \\
\hline & & PP-b-(PMMA $)_{x}$ & 5.0 & 220000 & 154 \\
\hline
\end{tabular}

a These metallocene-synthesized PPs are mostly isotactic, with a broad molecular weight distribution (typically $\bar{M}_{w} / \bar{M}_{n}=4-6$ ); the details of the polymerizations are published elsewhere (refs 2832). The neat/unmodified PP used is a commercial material with $\bar{M}_{\mathrm{w}}=580000$ and $\bar{M}_{\mathrm{w}} / \bar{M}_{\mathrm{n}}=3.49$.

details about the polymer synthesis and functionalization are given in length elsewhere. ${ }^{12,28-30}$ Even with such extremely low functionalization levels (1 mol \%), these polymers ${ }^{27}$ were readily miscible with dimethyldioctadecylammonium mmt (2C18-mmt). Obviously, the use of the dimethyldioctadecylammonium modification is chosen because 2 C18-mmt materials readily blend with the styrenic comonomers and their functionalized derivatives. ${ }^{31}$

The X-ray diffraction scans for the low $2 \theta$ values, where the basal reflection of the interlayer $d$ spacing appears, are shown in Figure 1 for functionalized-PP/ 2C18-mmt samples formed under static melt-intercalation conditions (i.e., the nanocomposites were formed by annealing the polymer/o-mmt physical mixture in a vacuum oven at $180^{\circ} \mathrm{C}$, unassisted by shear or solvent). There is a definite intercalated structure, for all the different functional groups that we tried, manifesting itself through an interlayer d-spacing increase of about $1 \mathrm{~nm}$ compared to that of the 2C18-mmt. Since these hybrids were formed in the absence of mechanical shear, this is direct evidence of sufficiently favorable thermodynamics for the nanocomposite formation. However, the XRD can only detect the periodically stacked $\mathrm{mmt}$ layers; for all these nanocomposites there also exists a large number of exfoliated layers as well, which can be directly observed by transmission electron microscopy (TEM). I n Figure 2 we show a bright-field TEM of the maleic anhydride functionalized polypropylene (PP-rMA) nanocomposite, containing 6 wt \% of 2C18-mmt. From the TEM it becomes clear that there are intercalated tactoids $(A)$ and disordered/exfoliated stacks of layers (B) coexisting in the nanocomposite structure. The intercalated structures are characterized by a parallel registry that gives rise to the XRD reflection of Figure 1 , whereas the disordered $\mathrm{mmt}$ formations have no 


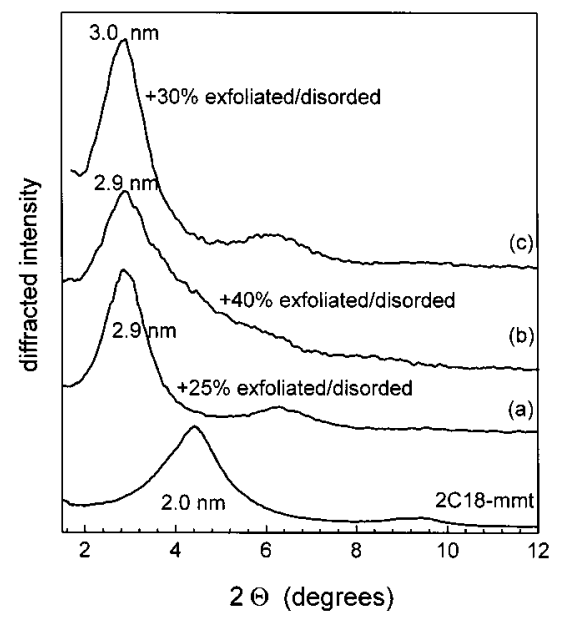

Figure 1. X-ray diffraction patterns of a dimethyldioctadecylammonium-modified montmorillonite (2C18-mmt) and all of the functionalized-PP/2C18-mmt nanocomposites. The functional groups used are (a) methylstyrene $1 \mathrm{~mol} \%$, (b) maleic anhydride $0.5 \mathrm{~mol} \%$, and (c) hydroxyl-containing styrene 0.5 mol \%. The characteristics of the random PP copolymers are tabulated in Table 1 . All the hybrids were formed by meltintercalation, and the content of exfoliated/disordered $\mathrm{mmt}$ layers mentioned for each system is quantified from image analysis of no less than 25 TEM pictures.

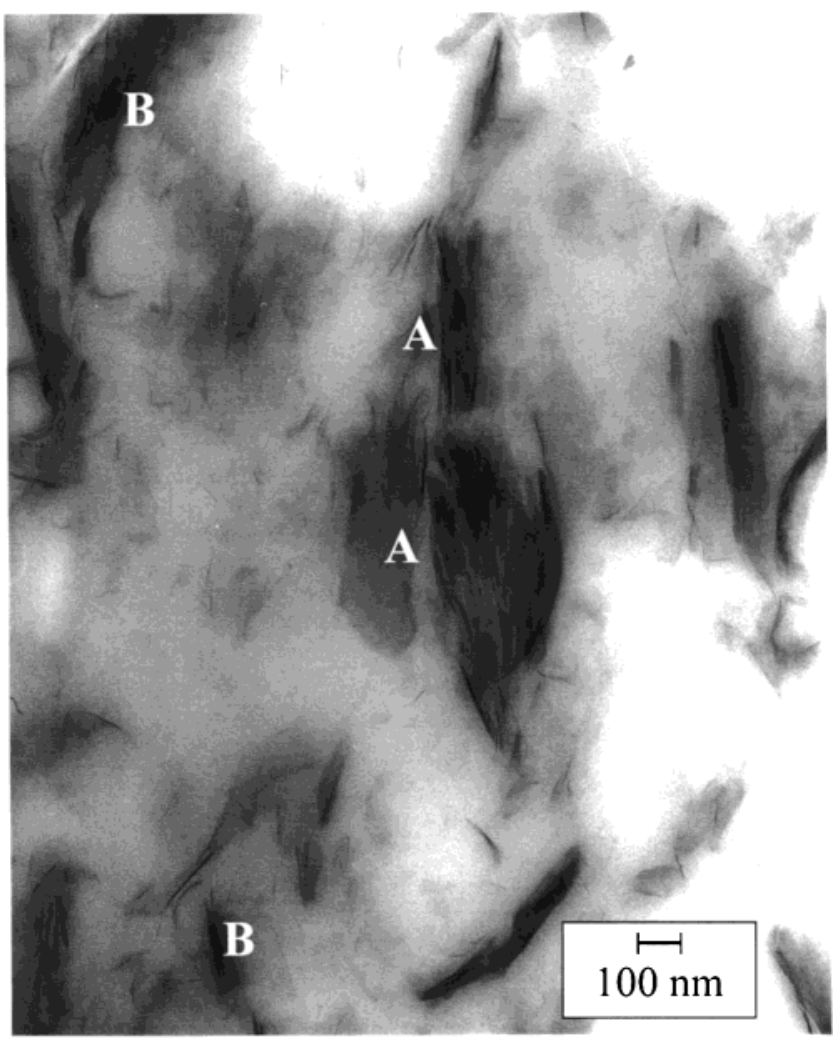

Figure 2. Characteristic bright-field TEM image of the functionalized-PP/2C18-mmt nanocomposite structure; a PP$\mathrm{r}-\mathrm{MA} / 6 \mathrm{wt} \% \mathrm{o}-\mathrm{mmt}$ is shown. There is a clear coexistence of intercalated $\mathrm{mmt}$ tactoids (A) and exfoliated/disordered $\mathrm{mmt}$ layers (B). Figure adapted from ref 11.

periodic stacking and thus remain XRD silent. This behavior is common for most polymer/mmt nanocomposites, ${ }^{3,4}$ and in accord with this general behavior, in our PP/mmt hybrids the larger-in lateral size-mmt layers create intercalated tactoids, whereas the smaller layers tend to exfoliate.

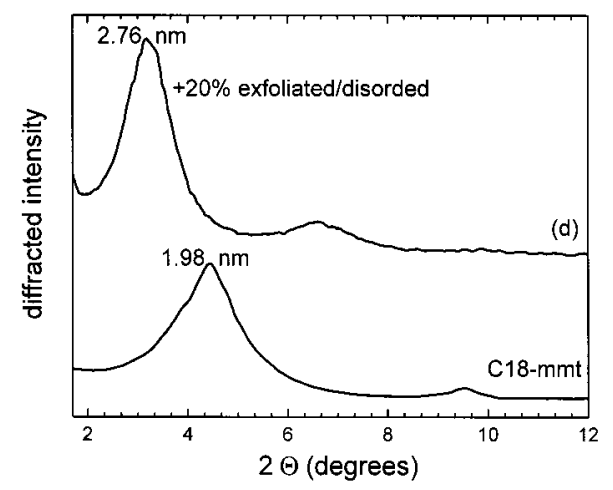

Figure 3. X-ray diffraction patterns of octadecylammoniummodified montmorillonite (C18-mmt) and of the PP-b-PMMA/ C18-mmt nanocomposite, formed by melt-intercalation. The amount of exfoliated/intercalated $\mathrm{mmt}$ layers denoted was estimated by image analysis of 32 TEM pictures.

Quantitative analysis of TEM images can be used to evaluate the percentage of silicate exfoliation. Namely, for each nanocomposite material, we capture 20-40 independent TEM images at $50 \mathrm{~K}$ magnification (a view of approximately $4 \times 5 \mu \mathrm{m}$ ). Subsequently, we use image analysis software to enumerate (a) the total number of layers seen edge-on, (b) the layers in stacks of more than 10 layers separated by $<3 \mathrm{~nm}$ (intercalated tactoids); and (c) bunches of up to 3 layers that are separated by more than $50 \mathrm{~nm}$ (exfoliated layers) or nonparallel layers separated by $<20 \mathrm{~nm}$ (disordered layers). The percentage of the last group is an estimation of the exfoliated and disordered structures in the system (this number is recorded in Figure 1 and onward). Admittedly, these definitions are ad hoc and 20-40 images cover a very small total area to carry high statistical significance; however, for the present study they were sufficient to contrast the different PP/mmt nanocomposite structures.

The functional groups added to the PP polymer do not have to be introduced in a random-copolymer fashion; alternatively, functional groups can be incorporated as a block, forming a PP diblock copolymer. To test this approach, we synthesized diblocks with poly(methyl methacrylate) (PP-b-PMMA) containing 1 and $5 \mathrm{~mol} \%$ PMMA. Their synthesis involved PP preparation by metallocene catalysis, hydroboranation of the olefinic chain end, and free radical polymerization of the PMMA block. ${ }^{32}$ Obviously, in this case it is necessary to use an organic modification for the mmt that will promote miscibility with PMMA, namely, a primary ammonium with an octadecyl tail (C18-mmt). The resulting composites contained approximately $20 \%$ of the layers in an exfoliated/disordered manner and the rest in an intercalated manner as shown in Figure 3.

Nanocomposite Formation through Fluoro-Organic Modified mmt. Our aim, when designing the functionalized PP above, was to render the polymer/mmt interactions more thermodynamically favorable than the surfactant/mmt interactions. Exactly the same effect can be achieved by introducing an organic modification in the mmt which is thermodynamically less favorable than the olefinic polypropylene; such a modification can be a semi-fluorinated surfactant. Expectedly, an o-mmt that is completely modified by fluorinated organic surfactants would be very difficult to obtain (since the 


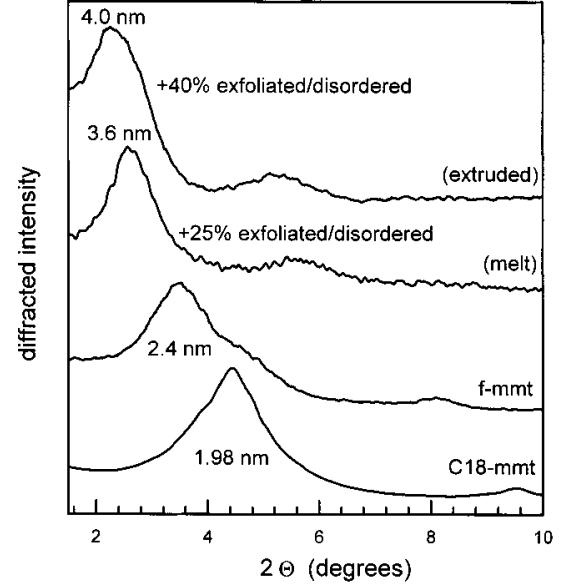

Figure 4. X-ray diffraction patterns of octadecylammoniummodified montmorillonite (C18-mmt) and C18-mmt modified by trichloro(1,1,2,2H-perfluoro octyl)silane (f-mmt). Also, the XRD of nanocomposites made with $\mathrm{f}$ - $\mathrm{mmt}$ and unfunctionalized PP $\left(\bar{M}_{\mathrm{w}}=580000\right)$, via melt-intercalation processing (melt) and through extrusion in a twin-screw extruder (extruded).

ultral ow surface tension surfactants will not exchange the alkali counterions under normal exchange conditions). Instead, a mixture of hydrogenated- and fluorinated-alkyl surfactants can be employed: Specifically, we first exchanged all the native mmt cations by octadecylammoniums, and subsequently we introduced a second semifluorinated alkyltrichlorosilane surfactant $\left(\mathrm{CF}_{3}-\left(\mathrm{CF}_{2}\right)_{5}-\left(\mathrm{CH}_{2}\right)_{2}-\mathrm{Si}-\mathrm{Cl}_{3}\right)$. This second surfactant is tethered to the $\mathrm{mmt}$ surface through a reaction of the trichlorosilane groups with hydroxyls in the cleavage plane of the mmt. ${ }^{12}$ The resulting o-mmt contains octadecylammoniums at full CEC and $\approx 60 \%$ additional semi-fluorinated surfactants (as quantified by TGA analysis). The XRD of the initial C18-mmt and the subsequent fluoro-organic $\mathrm{mmt}$ (f-mmt) are shown in Figure 4. In the same figure, we also show the XRD of unfunctionalized-PP/f-mmt nanocomposites, which were formed by melt-intercalation and by extrusion. The hybrid formed by melt-intercalation (i.e., by just annealing a physical mixture of polymer and $\mathrm{f}-\mathrm{mmt}$ ) reveals that there exist favorable thermodynamics for the nanocomposite formation [Figure 4 (melt)]. Moreover, the usage of mechanical shear [Figure 4 (extruded)] promotes further dispersion and exfoliation of the f-mmt layers.

At this point, we should note that this approach is qualitatively different than the "swelling agent" approach, as for example by Wolf et al. ${ }^{9}$ In such approaches an alkylammonium-exchanged montmorillonite is intercal ated by an organic "swelling agent", such as ethylene glycol, naphtha, or heptane (all with boiling points below the processing/extrusion temperature). ${ }^{9}$ Subsequently, the swollen organo-modified clay is compounded with PP in a twin-screw extruder at $250{ }^{\circ} \mathrm{C}$. At this processing temperature, the swelling agent evaporates, leading to the formation of a "nano"composite which is XRD silent. In principle, this is the same as the solution intercalation process, where a solvent is employed to mix the o-mmt with the polymer, and an exfoliated structure is trapped when evaporating the solvent. ${ }^{19}$ Such trapped structures are not thermodynamically stable and are not amiable to processing. Similar nanocomposites ${ }^{9}$ can also be obtained by copre- cipitation of PP and alkylammonium-mmt from a trichlorobenzene solution (as discussed in the following section).

Synopsis of the Synthetic Routes and the Nanocomposite Structures. Guided by simple thermodynamic arguments, we proposed two general synthetic routes to PP/organo-mmt nanocomposite formation and demonstrated their feasibility: (i) the first one implements "ordinary" organic modifications for the $\mathrm{mmt}^{33}$ and functionalized polypropylene polymers; (ii) alternatively, neat/unfunctionalized polypropylene can disperse mmt modified by a mixture of hydrogenated and semi-fluorinated surfactants. In both cases, polymer/ommt nanocomposites can be formed by melt-intercalation (i.e., unassisted by mechanical shear or organic solvents) Moreover, in both cases the resulting hybrid structures contain intercalated mmt tactoids in coexistence with exfoliated/disordered mmt layers. Where extrusion is used to form the PP/o-mmt nanocomposites, instead of melt-intercalation, further dispersion and exfoliation of the mmt layers is promoted.

We should mention that the second approach (employing f-mmt) is generally applicable for nonpolar polymers, such as polycarbonates, polyolefins, and polydienes. Some representative material properties of these PP/o-mmt hybrid materials are discussed in the following section.

\section{Materials Properties}

Stability of the Nanocomposite Structure. One of the first questions that should be addressed is whether the mmt dispersion (nanocomposite structure) is retained after high-temperature processing of the hybrid. To qualitatively assess the stability of the nanocomposite structure, we carried out the following experiment: We preci pitated hybrids from a co-suspension of polypropylene and o-mmt mixed in a common organic solvent (1,3,5-trichlorobenzene). As the solvent evaporates, the o-mmt layers become trapped in an almost exfoliated manner throughout the polymer matrix. ${ }^{19}$ Subsequently, we process these "hybrids" at 180 ${ }^{\circ} \mathrm{C}$ (i) by compression molding and (ii) by a commercial twin-head mixer (Brabender plasti-corder). Both these methods allow the polymer to melt and the trapped hybrid structure to relax. If the o-mmt dispersion is not thermodynamically stable, the layers will collapse in low d-spacing parallel stacks during the high-temperature processing, leading to a conventionally filled "macro" composite. However, if there exists a favorable free energy of the o-mmt/polymer mixing, the nanocomposite structure of Figures 1-4 will be largely recovered.

In Figures 5 and 6 we show the structure evolution under annealing-as revealed by XRD-for two kinds of systems: A neat-PP/dimethyldioctadecylammonium $\mathrm{mmt}$ (Figure 5 ), where PP and the alkyl-modified $\mathrm{mmt}$ are at theta conditions, and neat-PP/f-mmt (Figure 6a) PPMA/dimethyldioctadecylammonium mmt (Figure $6 b$ ), where there exist favorable thermodynamics for the o-mmt dispersion in the polymer.

All systems were precipitated-at time $0 \mathrm{~min}$-from a polymer/o-mmt co-suspension, and as cast they show an almost silent XRD pattern, which denotes that after the solvent was removed the mmt layers were trapped in an exfoliated or disordered dispersion. In the first 


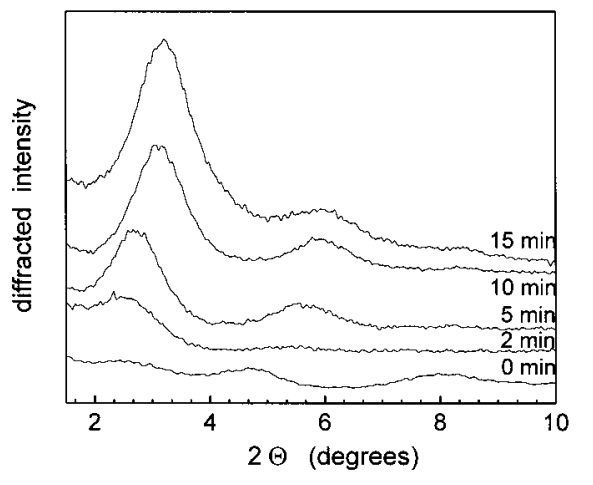

Figure 5. XRD time series of the structure stability/evolution of neat-PP/2C 18-mmt, under compression molding at $180{ }^{\circ} \mathrm{C} /$ 15 tons. In the precipitated "hybrids" the mmt layers are "trapped" in exfoliated/disordered dispersion (at time $0 \mathrm{~min}$ ), but quickly collapse toward intercalated and immiscible stacks.

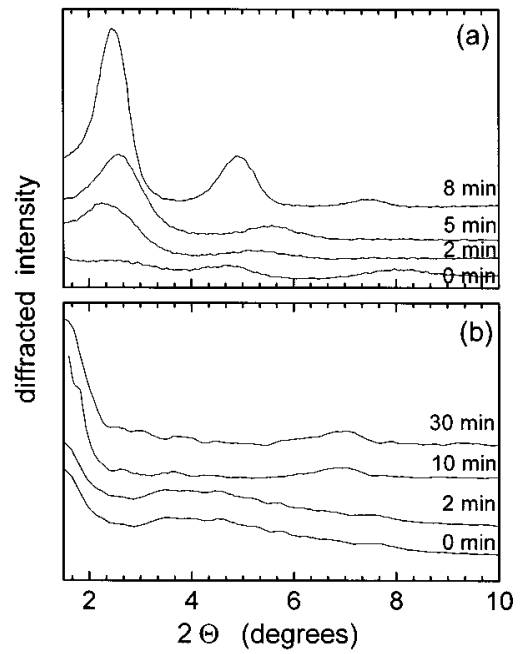

Figure 6. XRD time series of the structure stability/evolution of neat-PP/f-mmt in a twin-head mixer (a) and of PP-MA/2C18mmt under compression molding at $180{ }^{\circ} \mathrm{C}$ (b). In sharp contrast with Figure 5 , the trapped $\mathrm{mmt}$ layers are either stable under prolonged annealing (b) or under mechanical shear the intercalated/exfoliated structure of Figure 4 is recovered (a).

case, neat-PP/2C18-mmt was annealed in a compression molder at $180^{\circ} \mathrm{C}$ and 15 tons of load (Figure 5). For the neat-PP/2C18-mmt the dispersed layers coll lapse toward immiscible/intercalated structures within $10-15 \mathrm{~min}$ of annealing (as denoted by d-spacings of $2.4-3.1 \mathrm{~nm}$, Figure 5). F or the PP-r-MA however, even after $30 \mathrm{~min}$ of annealing at $180^{\circ} \mathrm{C}$ in the compression molder, there appears to be no intercalated peak (like that of Figure 1a); the trapped structure is maintained, even after prolonged annealing. This suggests that the MA groups have sufficiently strong interactions with the $\mathrm{mmt}$ to prevent the polymer from sliding away from the inorganic layers. Although this has been observed before for polymers with strong specific interactions with $\mathrm{mmt}$ (e.g., polymers that hydrogen bond to the silicates, such as poly(vinyl al cohol $)^{19}$ and nylon-6), it is striking that only $0.5 \mathrm{~mol} \%$ of MA can have the same effect.

The precipitated neat-PP/f-mmt hybrid was annealed in a Brabender mixer at $180^{\circ} \mathrm{C}$ (F igure 6a). As expected, the mechanical shear markedly reduces the time necessary for the structure recovery, and the structure of Figure 4 is recovered after 8 min of mixing. In sharp

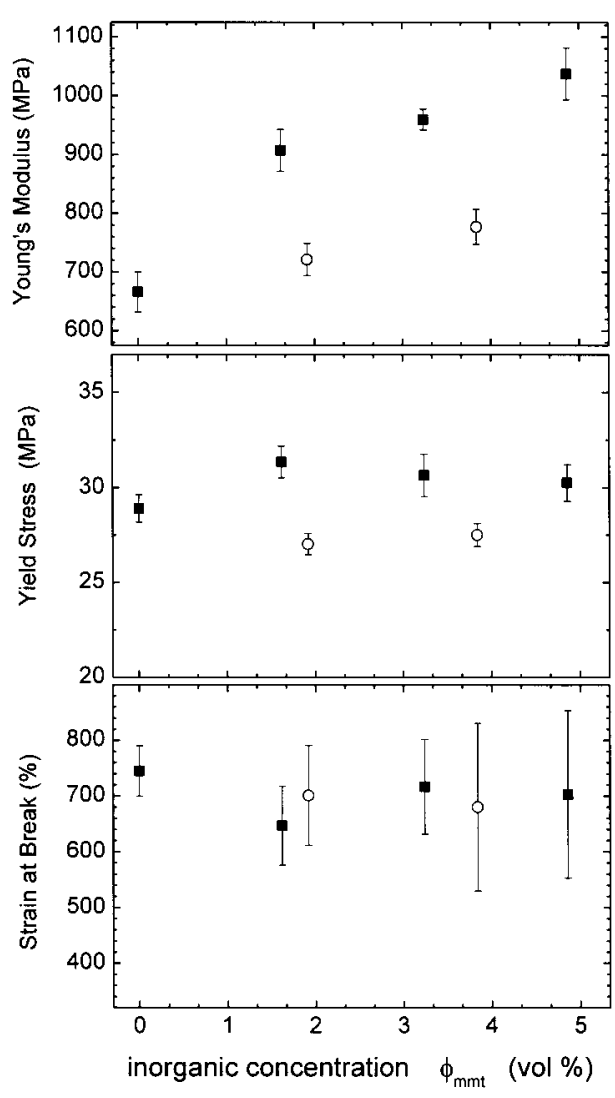

Figure 7. Tensile characterization of the PP/f-mmt nanocomposites ( $\boldsymbol{\square})$ by Instron. For comparison, conventionally filled PP/2C18-mmt "macro" composites are also shown (O). Figure adapted from ref 12.

contrast, precipitated systems of neat-PP and 2C18$\mathrm{mmt}$, also characterized by a silent XRD as cast, but even very moderate mixing in the Brabender $(2-5 \mathrm{~min}$ at $180^{\circ} \mathrm{C}$ ) resulted in an immiscible/intercalated structure with a wide XRD reflection extending from 1.8 to $2.7 \mathrm{~nm}$ in d-spacing.

Mechanical Properties. Most of the PP/mmt nanocomposites studies report tensile properties as a function of mmt content $(\phi \mathrm{mmt})$, characterized by Instron. ${ }^{5-12}$ As a typical example of thesetensile properties, we show in Figure 7 an Instron study of a neat-PP/f-mmt composite compared to a PP/2C18-mmt "conventional" composite (for these latter systems, the 2C18-mmt remains in tactoids, which are not intercalated or exfoliated by a polymer). The characteristic behavior for polymer/layered-inorganic nanocomposite materials ${ }^{3}$ is observed. Namely, there is a sharp increase of Young's modulus for very small inorganic loadings $\left(\phi_{\mathrm{mmt}}<3 \mathrm{wt}\right.$ $\%)$ followed by a much slower increase beyond $\phi_{\mathrm{mmt}} \simeq 4$ wt \%. With increasing $\phi_{\mathrm{mmt}}$, the yield stress does not change markedly compared to the neat-polymer value, and there is only a small decrease in the maximum strain at break. PP systems conventionally filled-no nanometer-level dispersion-by similar fillers (e.g., 2C18$\mathrm{mmt}$ ) do not exhibit as large increases in their tensile modulus (Figure 7). Similar improvements in mechanical properties can also be achieved by other layered particulate fillers; however, much higher filler loadings are required. For example, to obtain comparable tensile increases like the ones achieved by $\mathrm{mmt}$ dispersion (Figure 7), $30-60$ wt \% of talc or mica is needed. ${ }^{2}$ Obviously, as the polymer/inorganic adhesion is improv- 


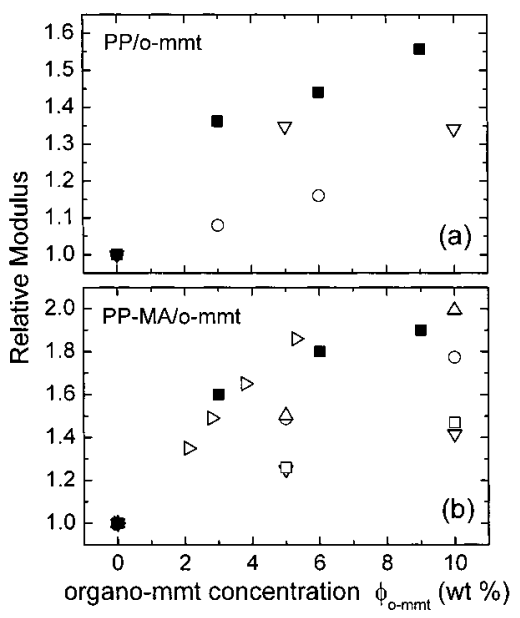

Figure 8. Relative Young's moduli of various PP nanocomposites, each normalized by Young's modulus of the respective PP. (a) neat-PP hybrids: with f-mmt ( $\mathbf{\square}$, ref 12$),$ C18-mmt $(\nabla$, ref 10), and 2C18-mmt (O, ref 12). (b) PP-r-MA/2C18-mmt nanocomposite ( $\mathbf{\square}$, ref 12 ) and PP hybrids with various PPMA pretreated o-mmt: C18-mmt (right triangle open, ref 7), C18-mmt $(O, \triangle$, ref 10$)$, and C8-mmt $(\nabla, \square$, ref 10$)$

ed-as for example when MA functional groups are incorporated in the polymer-the stresses are much more effectively transferred from the polymer matrix to the inorganic filler, and thus a higher increase in Young's modulus is expected (F igure 8). F or this reason, compared to neat-PP/mmt hybrids (Figure $8 \mathrm{a}$ ), the PP$\mathrm{MA} / \mathrm{mmt}$ 's are characterized by larger modulus increases for the same o-mmt loading (Figure 8b).

The tensile results obtained from this work are not affected by the nanocomposite processing since the nanocomposite structure does not change markedly with the processing conditions (i.e., whether the hybrid is formed by melt-intercalation or by extrusion). However, in the absence of favorable thermodynamics for the PP/ o-mmt miscibility, the hybrid structure and the tensile properties can be altered by the processing conditions (Figure 8b). In the work by Mülhaupt et al.,10 a systematic study of the dependencies on compatibilizer functionality and $\mathrm{mmt}$ organic modification revealed that considerable tensile enhancements can be achieved only where appropriate PP-MA "compatibilizers" are used to pretreat the o-mmt in conjunction with specific organic modification of the mmt. Similar materials under different processing conditions showed much smaller improvements in the mechanical properties. ${ }^{5-8}$

Heat Deflection Temperature. The mechanical reinforcement of PP due to the nanocomposite formation is not limited only to the tensile modulus increase. The nano-dispersion of mmt in the PP matrix also promotes a higher heat deflection temperature (HDT). In Table 2 we report the HDT ${ }^{34}$ of PP and of its nanocomposites based on $\mathrm{f}-\mathrm{mmt}$ and alkylammonium $\mathrm{mmt}$. In the case of neat-PP/f-mmt there is a marked increase of the HDT, from $109{ }^{\circ} \mathrm{C}$ for the neat polymer to $152^{\circ} \mathrm{C}$ for a 6 wt \% nanocomposite; beyond 6 wt \% of $\mathrm{f}-\mathrm{mmt}$ the HDT of the hybrid levels off. When the same neat-PP polymer is filled with alkylammonium-modified mmt, the HDT is also increased but to a smaller extent, reflecting the lower exfoliation level of the inorganic fillers. Moreover, in the latter case, there is a strong dependence of the HDT on the processing conditions during the composite
Table 2. Heat Deflection Temperatures (HDT (ref 34)) of PP/mmt Nanocomposites and the Respective Unfilled (Neat) PP

\begin{tabular}{lcc}
\hline \multirow{2}{*}{$\begin{array}{c}\text { organo-mmt } \\
\text { filler loading }\end{array}$} & \multicolumn{2}{c}{ HDT $\left({ }^{\circ} \mathrm{C}\right)$} \\
\cline { 2 - 3 }$\phi_{\text {o-mmt }}$ (wt \%) & neat-PP/f-mmt & neat-PP/alkyl-mmt \\
\hline 0 (neat PP) & $109 \pm 3$ & $109 \pm 3$ \\
3 & $144 \pm 5$ & $130 \pm 7^{\mathrm{a}}$ \\
6 & $152 \pm 5$ & $141 \pm 7^{\mathrm{b}}$ \\
9 & $153 \pm 5$ &
\end{tabular}

a C18-mmt filler, extruder processed. b 2 C18-mmt filler, twinhead mixer.

formation, for the same type of filler and the same loading. The increase of HDT due to mmt dispersion is a very important improvement for PP, not only from the application/industrial viewpoint but also because it is difficult to achieve similar HDT enhancements by chemical modification or reinforcement by other fillers. ${ }^{2}$

The improvement of the HDT originates from the better mechanical stability of the nanocomposite, compared to the neat-PP, rather than any increase of the polymer melting point. In all the PP/mmt hybrids studied, the melting temperature does not change markedly from that of the respective neat polymer. This is qualitatively different from the behavior of other polymers (e.g., nyl on-6) where the mmt layers stabilize a different crystalline phase than that found in the neat polymer, with a higher melting point and also higher HDT 15,16

Other Properties. Beyond the mechanical and HDT improvements, the nanocomposite formation results in concurrent enhancements of other materials properties. ${ }^{3}$ Because tensile properties can also be improved by other means, ${ }^{1,2}$ the highest promise for any potential uses of $\mathrm{PP} / \mathrm{mmt}$ nanocomposites do actually relate to these "other property" enhancements. In brief, we will discuss some examples that qualitatively set apart the PP/mmt nanocomposites from other conventional PP composites.

Albeit their micrometer lateral size, mmt's are just 1-nm thin. Thus, when single layers are dispersed in a polymer matrix, the resulting nanocomposite is optically clear in the visible region. In Figure 9 we present the UV/vis transmittance as a function of wavel ength, both for neat-PP/f-mmt and thick films of PP-r-MA/2C18$\mathrm{mmt}$. There is no marked decrease in the clarity due to the fillers (for o-mmt loadings up to $\phi=9$ wt \%). One has to load approximately 20 wt \% of 2C18-mmt in 3-mm-thick films of PP-r-MA before there develops haze observable by the naked eye. The loss of intensity in the UV region (for $\lambda<250 \mathrm{~nm}$ ) is mostly due to scattering by the mmt particles. When a neat-PP/ $6 \mathrm{wt}$ $\%$ f-mmt nanocomposite is compared to a conventionally filled neat-PP/3 wt \% C18-mmt, it is observed that these two materials have al most the same loss of intensity in the visible region (Figure 9). Despite the double inorganic loading in the PP/f-mmt system, due to the exfoliation of the mmt layers, these two materials have a comparable concentration of tactoids. This suggests that the intensity loss in the visible region is due to the intercalated/immiscible tactoids, whereas the exfoliated layers do not seem to contribute. Hence, achieving a fully exfoliated structure should lead to nanocomposites as optically clear as the initial polymers. ${ }^{19}$

Generally, polymer/silicate nanocomposites are characterized by a range of materials improvements, com- 


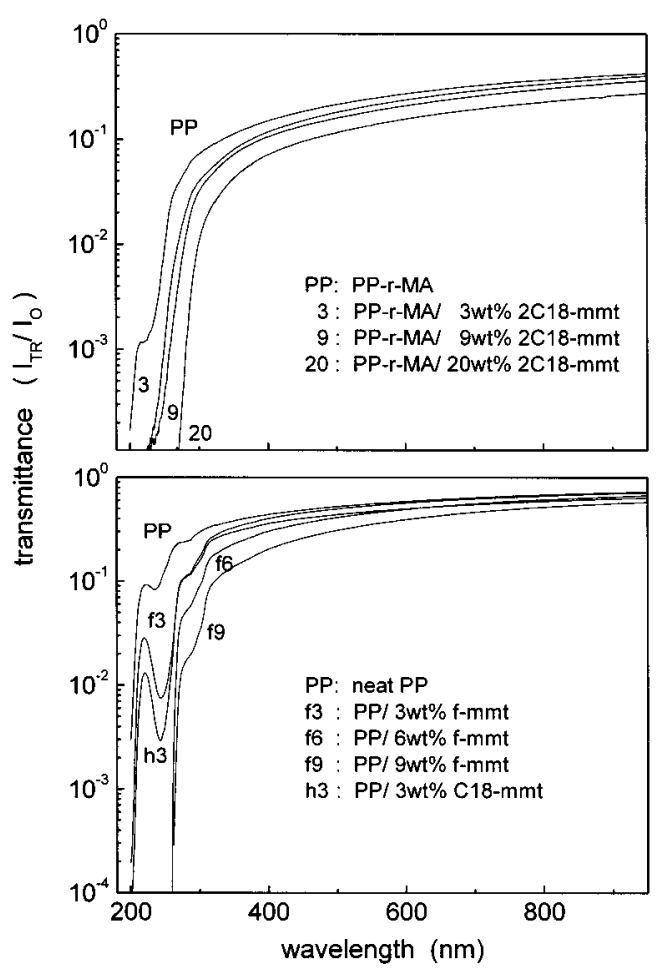

Figure 9. Optical clarity measurements through UV/vis transmittance for various PP/o-mmt nanocomposite films: (top) Thick films (3 mm) of PP-r-MA and its 2C18-mmt nanocomposites; in order for these nanocomposite films to become haze to the naked eye, the $2 \mathrm{C} 18-\mathrm{mmt}$ loading has to reach 20 wt \%. (bottom) Thin ( $1 \mathrm{~mm}$ ) films of unmodified PP and PP/f-mmt nanocomposites; in the visible region the PP/6 wt \% f-mmt nanocomposite has the same transmittance with a PP/3 wt \% C18-mmt "macro" composite, suggesting that the exfoliated layers do not contribute to any decrease in visible transmittance. From ref 12.

mon to most polymers, which originate from the nature of the inorganic filler and its nanometer-thin layered structure. ${ }^{3,4} \mathrm{PP} / \mathrm{mmt}$ nanocomposites also exhibit most of these "general" property enhancements. Namely, the permeability to liquids and gases is reduced to half when 4 wt \% of $\mathrm{mmt}$ is dispersed in the PP or the functionalized PPs, and at the same time, the solvent uptake decreases accordingly. ${ }^{12}$ Another property that is strongly affected by these inorganic fillers is a sharp increase of the scratch resistancefor very moderate loadings ( 1-5 wt \%) of o-mmt. Montmorillonite-based fillers also promote the flame retardancy of PP. In Figure 10 we show the mass loss rate during combustion, measured by cone calorimetry. For a PP-r-MA nanocomposite with 4 wt \% 2C18-mmt, there is a 75\% reduction in flammability compared to the unfilled polymer. ${ }^{18}$ This flameretardant character is traced to the response of a carbonaceous-char layer, which develops on the outer surface during combustion:18 This surface char has a high concentration of mmt layers and becomes an excellent insulator and a mass transport barrier (slowing the oxygen supply as well as the escape of the combustion products generated during decomposition). ${ }^{18}$

Finally, from a practical viewpoint, because only a few percent of inorganic fillers are needed in the PP/mmt nanocomposites, the resulting hybrids are lightweight. Moreover, where there exist favorable thermodynamics for PP/o-mmt miscibility, the organo-mmt can be incorporated in the final stages of polymer processing (e.g.,

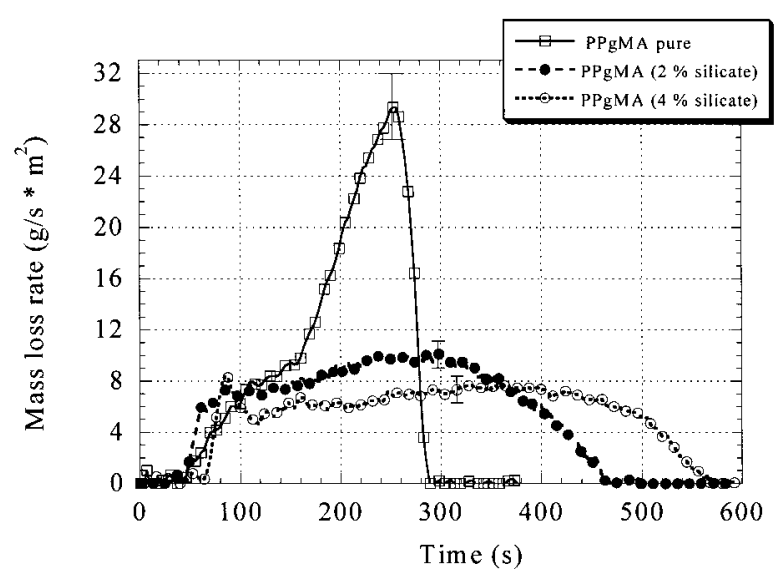

Figure 10. Mass loss rate during cone-calorimetry combustion of heat PP-r-MA and PP-r-MA/2C18-mmt nanocomposites ( 2 and $4 \mathrm{wt} \%$ in o-mmt). The nanocomposite formation strongly promotes flame retardancy. Figure from ref 18, where there also exists a detailed discussion of the general mechanisms responsible for the improvement in flammability in clayreinforced polymers.

extrusion, injection/compression molding) to obtain nanocomposite hybrids. Thus, $\mathrm{PP} / \mathrm{mmt}$ nanocomposites are amenable to most of the common processing techniques in today's industrial practices, which lowers the barriers toward commercialization for these nanocomposite hybrids. Additionally, o-mmt fillers can be used in conjunction with other PP reinforcements, such as fibers, thus combining the PP/mmt improvements and those from the fiber reinforcement in one composite material.

\section{Conclusions}

Synthesis of PP/o-mmt nanocomposites, with a coexisting intercalated and exfoliated structure, was realized in two ways: (i) by introducing functional groups in PP and using common alkylammonium montmorillonites and (ii) by using neat/unmodified PP and a semifluorinated surfactant modification for the mmt.

In the first case, the necessary level of PP functionalization is very low (random copolymers with $0.5 \mathrm{~mol}$ $\%$ of functionalized comonomers, or diblock copolymers with $1 \mathrm{~mol} \%$ of non-PP blocks, sufficed). Functional groups that promoted nanocomposite formation with 2C18-mmt included p-methylstyrene, maleic anhydride styrene, and hydroxyl-containing styrene; polypropylene-b-methyl-methacrylate diblocks also formed nanocomposites with C18-mmt. In the second case, a semifluorinated alkyltrichlorosilane was used to modify C18$\mathrm{mmt}$, rendering it miscible with neat/unfunctionalized PP. In all cases, nanocomposite formation was achieved by melt-intercalation (unassisted by mechanical shear or solvents), revealing sufficiently favorable thermodynamics of polymer/o-mmt mixing.

As is typical in most polymer/silicate nanocomposites, $\mathrm{PP} / \mathrm{mmt}$ hybrids exhibit concurrent improvements in several materials properties, for very moderate inorganic loadings (typically less than $6 \mathrm{wt} \%$ of $\mathrm{mmt}$ ). Enhanced properties include improved tensile characteristics, higher heat deflection temperature, high barrier properties, better scratch resistance, and increased flame retardancy. Because these PP/mmt nanocomposite matrixes are amenable to common processing tech- 
niques and can be further reinforced by traditional fillers, such as fibers, these hybrid materials hold high promise for new potential applications.

Acknowledgment. Partial support for this work was provided by NIST (Grant 70NANBOH0097) and NSF (DMR 9975624). Generous allocations of XRD were provided by the PSU metallurgy lab at the MS\&E department, and TEM was carried out in the electron microscopy facility of the Materials Characterization Lab at the Penn State MRI .

\section{References}

(1) Karger-Kocsis, J., Ed. Polypropylene: Structure, Blends and Composites, vol. 3; Chapman and Hall: London, 1995.

(2) Karian, H. G., Ed. Handbook of Polypropyleneand Polypropylene Composites; Marcel Dekker: New York, 1999.

(3) Alexandre, M.; Dubois, P. Mater. Sci., Eng. R: Reports 2000, $28,1$.

(4) Giannelis, E. P.; Krishnamoorti, R. K.; Manias, E. Adv. Polym Sci. 1998, 138, 107-148.

(5) Kato, M.; Usuki, A.; Okada, A. J . Appl. Polym. Sci. 1997, 66, $1781-1785$.

(6) Kawasumi, M.; Hasegawa, N.; Kato, M.; Usuki, A.; Okada, A. Macromol ecules 1997, 30, 6333-6338.

(7) Hasegawa, N.; Kawasumi, M.; Kato, M.; Usuki, A.; Okada, A. J. Appl. Polym. Sci. 1998, 67, 87-92.

(8) Oya, A.; Kurokawa, Y.; Yasuda, H. J . Mater. Sci. 2000, 35, 10451050

(9) Wolf, D.; Fuchs, A.; Wagenknecht, U.; Kretzschmar, B.; J ehnichen, D.; Häussler, L. Proceedings of the Eurofiller' 99, LyonVilleurbanne 1999; pp 6-9.

(10) Reichert, P.; Nitz, H.; Klinke, S.; Brandsch, R.; Thomann, R.; Mülhaupt, R. Macromol. Mater. Eng. 2000, 275, 8-17.

(11) Manias, E.; Touny, A.; Wu, L.; Lu, B.; Strawhecker, K.; Gilman J. W.; Chung, T. C. Polym. Mater. Sci., Eng. 2000, 82, 282

(12) Manias, E.; Touny, A.;Wu, L.; Lu, B.; Chung, T. C. Macromolecules, in press.

(13) Theng, B. K. G. Formation and properties of clay-polymer complexes; Elsevier: Amsterdam, 1979.

(14) Theng, B. K. G. Chemistry of clay-organic reactions; Wiley: New York, 1974.

(15) Kojima, Y.; Usuki, A.; Kawasumi, M.; Okada, A.; Fukushima, Y.; Kurauchi, T. T.; Kamigaito. O. J . Mater. Res. 1993, 8, 11791185.

(16) Kojima, Y.; Usuki, A.; Kawasumi, M.; Okada, A.; Kurauchi, T. T.; Kamigaito. O. J . Polym. Sci. Part A: Polym. Chem. 1993 $31,983$.

(17) Vaia, R. A; Ishii, H.; Giannelis, E. P. Chem. Mater. 1993, 5 , 1694-1696.
(18) Gilman, J . W.; J ackson, C. L.; Morgan, A. B.; Harris, R.; Manias, E.: Giannelis, E. P.; Wuthenow, M.; Hilton, D.; Philips. S. H. Chem. Mater. 2000, 12, 1866-1873.

(19) Strawhecker, K.; Manias, E. Chem. Mater. 2000, 12, 2943-2949.

(20) Xu, R.; Manias, E.; Snyder, A.J .; Runt, J . Macromol ecules 2001 34, 337-339.

(21) Vaia, R. A; Vasudevan, S.; Krawiec, W.; Scanlon, L. G.; Giannelis, E. P. Adv. Mater. 1995, 7, 154.

(22) Vaia, R. A; Giannelis, E. P. Macromolecules 1997, 30, 79907999.

(23) Vaia, R. A; Giannelis, E. P. Macromolecules 1997, 30, 80008009.

(24) Balazs, A. C.; Singh, C.; Zhulina, E. Macromolecules 1998, 31, 8370-8381.

(25) Confinement of the polymer inside the interlayers results in a decrease in the conformational entropy of the polymer chains. However, this entropic penalty of polymer confinement may be compensated in part by the increased conformational freedom of the tethered surfactant chains that are located in a less confined environment as the layers separate. ${ }^{22-24}$

(26) Excess enthalpy in the sense of polymer/mmt interactions being more favorable than the alkyl-surfactant/mmt interactions.

(27) The yield of conversion from p-MS to groups containing either $\mathrm{MA}$ or $\mathrm{OH}$ is approxiamtely $50 \%$. This means that our functionalized PP contained either $1 \mathrm{~mol} \%$ of $\mathrm{p}-\mathrm{MS}$ (initial polymer) or $0.5 \mathrm{~mol} \%$ of $\mathrm{p}-\mathrm{MS}$ plus $0.5 \mathrm{~mol} \% \mathrm{MA} / \mathrm{OH}$.

(28) Lu, B.; Chung, T. C. J . Polym. Sci. A: Polym. Chem. 2000, 38 1337-1343.

(29) Lu, H. L.; Hong, S.; Chung, T. C. J . Polym. Sci. A: Polym. Chem 1999, 37, 2795-2802.

(30) Lu, H. L.; Hong, S.; Chung, T. C. Macromolecules 1998, 31, 2028-2034.

(31) Manias, E.; Chen, H.; Krishnamoorti, R. K.; Genzer, J . ; Kramer E. J . ; Giannelis, E. P. Macromolecules 2000, 33, 7955-7966.

(32) Chung, T. C.; Lu, H. L.; J ankivul, W. Polymer 1997, 38, 14951502.

(33) The dimethyldioctadecylammonium modification for the mmt is comparable with the commercially available dimethylditallowammonium surfactants commonly used as organic modifications for montmorillonites and bentonites (e.g. Southern Clay Products: Cloisite 20A.; Nanocor: I42E.; Rheox: B34). Meltintercalation of our functionalized PP with these commercial organo-clay materials gave the same structures as those in Figure 1. Moreover, there also exist commercially available o-mmt's (such as the Nanocor I30E) that can be further modified by fluorinated trichlorosilanes so as to obtain neat-PP/f-mmt nanocomposites like the ones shown in Figure 4.

(34) ASTM standard. D 648-98c, "Standard Test Methods for Deflection Temperature of Plastics Under Flexural Load in the Edgewise Position" Annual Book of ASTM Standards; ASTM: Philadelphia, April 1999

CM0110627 